\title{
Molecular epidemiology of foot and mouth disease, bluetongue and pest de petites ruminants in Algeria: Historical perspective, diagnosis and control
}

\author{
Moustafa KARDJADJ ${ }^{1 *}$ and Pam Dachung LUKA ${ }^{2}$ \\ ${ }^{1}$ Ecole Supérieure en Science de l'Aliments (ESSA), Algiers, Algeria. \\ ${ }^{2}$ Biotechnology Division, National Veterinary Research Institute, Plateau State, Nigeria.
}

Received 15 September 2016, Accepted 13 October, 2016

\begin{abstract}
Molecular tools have become an increasingly important part of studying the epidemiology of infectious agents. These tools have allowed the aetiological agent within a population to be diagnosed rapidly with a greater degree of efficiency and accuracy than conventional diagnostic tools. They have enhanced understanding into the pathogenicity and virulence of the aetiological agent and subsequent deployment of appropriate control strategies. This paper reviews the contribution of molecular epidemiology to the diagnosis and control of some animal diseases such as foot and mouth disease (FMD), buetongue and peste des petites ruminants (PPR) in Algeria. Molecular epidemiology has helped in the characterization of FMDV type $O$ circulating in Algerian cattle in 1999 and in 2014; in 1999, the sequencing analysis showed that the Algerian viruses belong to the West-African topotype with $99 \%$ similarity to a strain isolated in Côte d'Ivoire. In 2014, the virus was identified as $0 / \mathrm{ME} / \mathrm{SA} / \mathrm{Ind}-2001 \mathrm{~d}$ lineage which was $99.69 \%$ identical to the field strains isolated from earlier Tunisian outbreaks. In a related development, two episodes of bluetongue outbreaks were reported in Algeria; the first with serotype II in $\mathbf{2 0 0 0}$ that showed no significant difference with the Tunisian strain reported two months earlier and the second episode involving serotype I epidemiologically linked to South Africa (with $94.3 \%$ not similarity) indicating an origin from sub-Saharan Africa. Molecular techniques have also described the PPRV strain implicated in an outbreak in Ghardaïa district, in the centre of Algeria. The strain clustered with lineage IV of PPRV and shared 97 to $99 \%$ similarity with the strain implicated in neighboring Morocco and Tunisia.
\end{abstract}

Key words: Algeria, control, diagnosis, molecular epidemiology, Foot and Mouth Disease (FMD), bluetongue (BT), Peste des Petites Ruminants (PPR).

\section{INTRODUCTION}

Molecular tools have increasingly become an integral part of studying the epidemiology of infectious agents globally

${ }^{*}$ Corresponding author. E-mail: drkardjadj@live.fr. Tel: +213796915906.

Author(s) agree that this article remains permanently open access under the terms of the Creative Commons Attribution License 4.0 International License 
(Van Belkum et al., 2007). Epidemiology, the study of factors determining the occurrence of disease/health outcome in a population, aims to describe the health status of a population, aetiology of disease, predict disease occurrence and to help control the distribution of disease. Molecular biology provides one of the many diagnostic tools that can be utilized to strengthen understanding of the epidemiology of a disease, both infectious and non-infectious (Grenfell et al., 2004). Molecular epidemiology has recently been defined as "a science that focuses on the contribution of potential genetic and environmental risk factors, identified at the molecular level, to the aetiology, distribution and prevention of disease within families and across populations" (Maslow et al., 1993). This science has emerged through integration of the disciplines of epidemiology and molecular biology. Molecular tools often provide additional dimension to the epidemiology of disease that would otherwise be impossible using conventional diagnostic tools. The objectives of molecular epidemiology for the study of infectious diseases include: (i) descriptive and analytical studies to evaluate host/environmental interactions in disease, and (ii) the development of strategies for the control of bacterial, parasitic and viral disorders through molecular diagnosis (Grenfell et al., 2004; Van Belkum et al., 2007).

Recently, the use of molecular tools has been helpful in unraveling the epidemiological relationships between infectious diseases, particularly emerging and reemerging transboundary animal diseases (TADs). These emerging TADs are those that are newly recognized and/or newly evolved or that have occurred previously but show an increase in incidence or expansion in geographical, host or vector range (Thompson et al., 1998; Riley, 2004). Multiple host species are sometimes involved in the transmission of diseases including wildlife, companion animals, livestock and fish, as well as several human behavioral risk factors that increase exposure to diseases. An integral part of prevention and control of these diseases is based on early and rapid detection, epidemiological investigation, multidisciplinary collaboration and the development of advanced diagnosis and surveillance tools, including the use of molecular biological methods such as PCR and phylogenetic analysis (Levin et al., 1999; Riley, 2004).

These molecular tools have been helpful inaccurately diagnosing diseases within a population with greater sensitivity and specificity compared to conventional methods. It has contributed to the increased understanding of the pathogenicity and virulence of aetiological agents, identified genes of the aetiological agent that are responsible for virulence, drug resistance, evasion of the immune system, provided information on the population structure, taxonomy, source and transmission dynamics of aetiological agents and identified genes that increase host susceptibility to disease (Thompson et al., 1998; Levin et al., 1999).
Adequate application of molecular epidemiological principles requires a working knowledge of both molecular biological and epidemiological methods. This review describe how the application of molecular tools can help elucidate aspects of the epidemiology and transmission patterns of foot and mouth disease (FMD), bluetongue (BT) and pest de petites ruminants (PPR) in Algeria using data available in research papers and reports of international organizations and databases (OIE, FAO).

Algeria is the largest country in Africa. It is located between latitudes $19^{\circ}$ and $37^{\circ} \mathrm{N}$ and longitudes $9^{\circ} \mathrm{W}$ and $12^{\circ} \mathrm{E}$. It is bounded by the Mediterranean Sea to the north, Tunisia to the east, Morocco to the west, Mali and Niger to the south. It has a long coastline at the Mediterranean Sea $(1600 \mathrm{~km})$; Most of the coastal areas (northern region) are hilly and sometimes even mountainous. South of the northern region is a steppe; farther south, there is the Sahara Desert. For reasons of animal health, transportation of animals is forbidden between Sahara and Northern Algeria. Administratively, Algeria is divided into 48 districts with a superficies of $2,147,570 \mathrm{~km}^{2}$ and with about 40 million people. More than $80 \%$ of the people live in coastal areas (MADR, 2015).

In Algeria, livestock farming represents a significant financial income of an important part of the Algerian population with $\approx 2$ million cattle and 31 million small ruminants all reared under traditional extensive husbandry system, although intensive husbandry systems have recently been introduced in the country. By virtue of its geographical location and its borders with the North African and Sahel countries, Algeria is vulnerable to several trans-boundary diseases, including FMD, BT and PPR (MADR, 2015).

\section{MOLECULAR EPIDEMIOLOGY OF FMD IN ALGERIA}

\section{The 1999 epidemic}

The livestock population (cattle, sheep and goat) at risk for FMD during the 1999 epidemic in Algeria was approximately 78 million heads because most of these livestock were not vaccinated against any of the circulating FMD serotype. Subsequently, on the $20^{\text {th }}$ and $21^{\text {st }}$ February 1999, two cases of foot-and-mouth disease (FMD) were suspected in cattle in Algiers district, Algeria (FAO, 1999). The vesicular material collected and sent to the World Reference Laboratory (WRL) for FMD in Pirbright confirmed type $O$ circulating and OIE and FAO were informed appropriately (FAO, 1999). Sequence analysis of the virus revealed a genetically different type $O$ virus from the strains that were currently circulating in the Meddle East and North Africa (MENA) between 1989 and 1997. Sequence analysis showed that the Algerian viruses (O/ALG/1/99) belong to the West-African 
topotype with $99 \%$ similarity to a strain isolated in Côte d'Ivoire (O/CIV/8/99) and Guinea (O/GNA/6/99) in 1999 (Samuel et al., 1999; Samuel and Knowles, 2001).

However, this confirmed the suspicion about the origin of the disease. Indeed, zebu cattle introduced illegally across the Algerian southern frontiers during the month of February 1999, were intercepted within the southern borders of the country. At the time of capture, these zebu cattle did not present any clinical signs of FMD (Samuel and Knowles, 2001). However, their presence demonstrated that transboundary animal movements took place on the southern frontier with Niger and Mali which are endemic for FMD. From the beginning of the epizootic up to the 22th June 1999; 179 outbreaks were recorded from 36 districts out of 48 infected by the disease. On the 22nd February 1999, cases of FMD were declared in Souk-Ahras district $50 \mathrm{~km}$ from the Tunisian border and Tlemcen at the west border of the country with Morocco (FAO, 1999).

Following this FMD epidemic, an appeal for vigilance was launched throughout national territories in Algeria, Tunisia and Morocco with active surveillance in all farms and all veterinary professionals were mobilized and biosafety measures observed. The media was used to sensitize and disseminate information on the benefits of farmer's participation in disease prevention and control program to protect their livestock. All cattle within affected farms were destroyed and the owners compensated, along with intensification of surveillance on a $10 \mathrm{~km}$ radius from the diseased area. The vaccine used for this campaign contains the $\mathrm{O}$ Manisa strain in accordance with the recommendations of the WRL, Pirbright (FAO, 1999; Thomson, 2002).

\section{The 2014 to 2015 epidemics}

On 24 April, 2014, two cows with clinical signs suggestive of FMD were reported in Nabeul district, Tunisia (OIE, 2016). The disease was confirmed by real-time RT-PCR and the phylogenetic analysis identified topotype O/ME$\mathrm{SA} /$ Ind $2001 \mathrm{~d}$ which is closely related $(99 \%)$ to recent viruses isolates from Libya (LIB/2/2013 and Saudi Arabia (SAU/3/2013). OIE report suggested that the source of the outbreak was due to the illegal movement of animals from Libya. During the first month (May, 2014), 32 new outbreaks were reported in domestic sheep, goats and cattle in 11 different districts. In June, new cases were subsequently declared in Jendouba districts $50 \mathrm{~km}$ to the western border of the country with Algeria (OIE, 2016).

According to OIE report, the source of the outbreak was due to illegal movements of ruminants from Libya into other Maghreb countries (OIE, 2016). Oueslati (2012) reported that most uncontrolled movements of ruminants in Maghreb countries occurred by land transport; given that the region is characterized by very long borders that stretch into the desert; $460 \mathrm{~km}$ (Tunisia-Libya), $520 \mathrm{~km}$
(Tunisia-Algeria) and $1040 \mathrm{~km}$ (Algeria-Libya). This remained a major constraint for border control services; the flow of uncontrolled movements of animal across the border that occurs along the east-west axis between Maghreb countries is difficult to estimate and depends on several factors (price changes, religious festivities, etc.). Furthermore, political unrest in Libya increased the potential risk of transboundary diseases spreading into neighboring border countries especially Tunisia and Algeria; this is mainly driven by the disruption of public health services, insecurity and massive displacement of refugees across the borders (OIE, 2016).

On the 23rd of July, 2014, FMD outbreak was detected in Setif district at the East of the Algeria, $260 \mathrm{~km}$ from border with Tunisia. The first outbreak occurred on a fattening cattle farm, the source of the outbreak was attributed to illegal introduction of animals from Tunisia. Clinical signs of the disease at the time of diagnosis included fever, blisters, lameness and mammary lesions (OIE, 2016). Samples were forwarded to the Experimental Zooprophylactic Institute (IZSLER), Brescia (OIE's Reference Laboratory) and the virus isolated and identified as O/ME-SA/Ind-2001d lineage with identity of 99.69 and $99.37 \%$, to field strains O/TUN/1031/2014 and O/TUN/1054/2014, respectively isolated during the current outbreaks in Tunisia (WRLFMD, 2016). Outbreaks were reported in the first week in six different districts. Again the second week also witness new outbreaks in 13 new districts, and by the end of August more than 350 outbreaks were recorded since the epidemic started in 33 different districts. Cases were subsequently declared in Oran districts $160 \mathrm{~km}$ to the western border of the country with Morocco. All the cases recorded were from cattle and there were no clinical signs of FMD in small ruminants. However, in March 2015, twelve FMD outbreaks involving sheep were reported in El Bayadh and El Oued districts ending nearly five months of absence of the disease in the Algeria (OIE, 2016).

Following the FMD epidemic in Tunisia in April 2014, several measures were implemented in Algeria (OIE, 2016)]. Crisis cells centres at national and regional levels were instituted; disinfection of vehicles leaving affected or suspected district; vaccination points of susceptible species at the entrance of livestock markets, Peri-focal vaccination in $5 \mathrm{~km}$ radius; epidemiological investigation to determine the origin of the infection; closing of livestock markets, ban on movement of animals within the infected districts. Treatment of animals was not carried out. In the affected farms, all cattle infected were destroyed and their owners compensated. Further control measures were; stamping out, screening, vaccination in response to outbreaks, disinfection of infected premises/establishments. Vaccination campaign throughout Algeria and Tunisia was performed. The vaccination was carried out with the same vaccine $(O$ Manisa) used in Libya and Tunisia. In Algeria, the 
vaccination campaign rate by June 2014 was $85 \%$ in cattle, however and despite the vaccination coverage FMD outbreaks has occurred in Algeria with a wide spared in all the country (OIE, 2016; WRLFMD, 2016).

A summary of vaccine matching data generated at the WRLFMD for representative member countries for the O/ME-SA/Ind2001d lineage showed results for 22 field virus samples sent to WRLFMD to contain data for viruses from Algeria and Tunisia. In general, three vaccine antigens (O/TUR/5/09, O-3039 and O/TAW/98) were matched against these viruses, while the in-vitro test indicated a poorer match for O-Manisa and O-BFS; the vaccine strains used in Algeria and Tunisia. Arguably, this may be the reason why the FMD epidemic continued in Tunisia and Algeria despite the vaccination efforts applied by the two countries, until August 2014 where the vaccine strain O/TUR/5/09 was used that allowed the control and resolution of the episodes (WRLFMD, 2016).

\section{MOLECULAR EPIDEMIOLOGY OF BT IN ALGERIA}

\section{The 2000 epidemics}

For the first time in history, Algeria reported 28 outbreaks of BT between July and September 2000 in the northeastern part of the country. Circulation of BTV serotype 2 was confirmed by the Institute of Animal Health (IAH) in Pirbright (Hamida, 2000). The disease spread after the first cases were reported to affect 24 localities in the district of Jijel. Of the 21,175 susceptible sheep, 2,661 $(12.6 \%)$ were clinically affected. The disease continued to spread and by the end of the epidemic, six more districts in the eastern and central parts of the country were also affected (Skikda: 1,277 cases; Souk Ahras: 430 cases; Annaba: 500 cases; Guelma: 2,871 cases; Oum El Bouaghi: 5 cases; Tebessa: 35 cases; and Jijel: 18 cases) (OIE, 2016). Molecular studies comparing genomic segments 2 and 7 of the virus isolated in Algeria to those isolate in Tunisia in May 2000 showed no significant difference between them (segment 2: 99.4\% homology; segment 7: 100\% homology). Therefore, the two isolates were probably of the same origin (Ben Fredj et al., 2003).

Since the incursion of BT into Tunisia in June 2000, the Algerian veterinary authorities implemented surveillance programmes to control BT in the country and to detect new clinical cases by serological diagnosis and determine the presence and distribution of known vectors of the disease. Once BT had been confirmed in Tunisia, the national veterinary authorities implemented a series of control measures also. Premises where outbreaks were recorded, flocks were isolated and dead animals buried. Sick animals, animal holdings and surrounding areas were sprayed with insecticide. Surveillance for the detection of new clinical cases in the nearby flocks was initiated but no vaccination was carried out (Hamida, 2000).

\section{The 2006 to 2007 epidemics}

In July 2006, an outbreak of BTV serotype 1 occurred in central Algeria and a total of 28 outbreaks were officially confirmed in the whole of Algeria between July 19 and August 30, 2006. BTV-1 was isolated in two regions, and was clinically identified and confirmed by real-time PCR with high level of seroconversion. A total of 5245 sheep were considered as susceptible with 263 cases. Thirty-six (36) deaths were reported during the outbreak with an apparent morbidity and mortality rates of 5.01 and $0.69 \%$, respectively (Cêtre-Sossah et al., 2011; Madani et al., 2011).

BTV was isolated from three different samples, derived from two different provinces (El Bayadh and Médéa). Amplification and sequencing of different genome segments (Seg-2, -7, -8, -9 and -10) was successfully carried out for identification of three isolates (CêtreSossah et al., 2011). In depth analysis of BTV-1 isolates from around the world, have identified two introductions of BTV-1 into the Mediterranean region, in 2001 and 2006 with a separate introduction of an African strain of BTV-1 into Oman in 2009 (www.reoviridae.org/dsRNA_virus_proteins/ReolD/BTVmol-epidem.htm):

1. BTV-1 in Greece in 2001 was thought to have entered Europe from the east, possibly via Turkey, although there was no previous evidence of BTV-1 during a serological survey of Turkey in the early 1980s. Unlike other western strains of BTV-1, the Greek type 1 strain did not spread to other European countries (Mellor et al., 2008).

2. In 2006, BTV-1 appeared in Algeria, and then spread to Morocco. Analyses of the Seg-2 from BTV strains belonging to the outbreak that started in Algeria during 2006 were (collectively) most closely related to the reference strain of BTV-1 from South Africa (94.3\% not similarity) indicating an origin from sub-Saharan Africa. The Algerian strains were only distantly related to BTV-1 from Greece 2001 (GRE2001/01) or BTV-1 from India (IND1992/01) (74.4 and $74.9 \%$ identity, respectively) although the Indian and Greek isolates were themselves closely related (95.9\% not similarity). BTV-1 has also been isolated in South Africa, India, China, Honduras, USA and Australia giving it a global distribution (CêtreSossah et al., 2011; Madani et al., 2011).

In 2007, BTV-1 belonging to the same western virus lineage was again identified, North Africa, in Algeria, Tunisia, Libya and Morocco and in the south of the Iberian Peninsula (Spain, Portugal and Gibraltar). This movement into Europe may have been caused by windborne movement of adult Culicoides in a similar manner to the movement of BTV-4 from Morocco to lberia during 2003 (Maan et al., 2009).

In 2007, the Algerian BTV-1 spread northwards to France and by late 2008, had arrived on the northern coast of the country becoming established in 2009, and 
threatening the UK. These movements provided the first overlap between the northern European outbreak of BTV8 and another BTV strain/serotype and are likely to provide opportunities for genome segment exchange, potentially leading to the generation of novel reassortant viruses (Maan et al., 2009).

Bluetongue is of significant veterinary concern to small ruminant producers, wildlife managers and veterinary diagnosticians because of the frequent occurrence of outbreaks among domestic and wild ruminants in geographical regions previously known to be BT-free (Saegerman et al., 2008). Recently Kardjadj et al. (2016) reported that the serological evidence of BTV exposure in Algeria was observed in 37 (17 cattle and 20 small ruminants herds) out of 225 herds accounting for $16.44 \%$ (95\% Cl 9.42-23.46) herd seroprevalence; $21.3 \%(95 \%$ $\mathrm{Cl} 17.1-25.6)$ for cattle and $13.33 \%(95 \% \mathrm{Cl} 9.86-16.8)$ for small ruminants. At the individual level, our results reveal an individual seroprevalence of $6.96 \%(200 / 2871)$; $13.7 \%(62 / 450)$ for cattle and $5.70 \%$ (138/2421) for small ruminants.

\section{MOLECULAR EPIDEMIOLOGY OF PPR IN ALGERIA}

Similarly, following the PPR epizootiology in Morocco in 2008 where 257 outbreaks were recorded with severe economic losses (FAO, 2009), Sghaier et al. (2014) in Tunisia reported a PPRV strain belonging to lineage IV and genetically related to those isolated in Morocco. In 2011, PPR was reported in Sahrawi refugee camps in Tindouf district, at the south western border of Algeria with Western Sahara, Mauritania, and Morocco and the sequence analysis clustered the circulating virus under lineage IV of PPRV (De Nardi et al., 2012). A year later, Kardjadj et al. (2015a) described the first serological and molecular typing of the PPRV strain implicated in an outbreak in Ghardaïa district, in the center of Algeria. The strain was clustered with lineage IV of PPRV and shared between 97 to $99 \%$ similarity with the strain implicated in neighboring Morocco and Tunisia.

Regular PPRV epizootic activity across the tropical and sub-tropical areas of North Africa has resulted in the spread of the disease into uninfected areas within the continent. The recent results from the Food and Agriculture Organization (FAO)-funded project "Toward a harmonized strategy for the control of Peste des Petits Ruminants in North Africa FAO Project (TCP/RAB/3302) provide insights into the situation on PPR in the Northern African countries (Algeria, Egypt, Libya, Morocco, Mauritania and Tunisia) updated up to 2012 to 2013. The results of this project show a high herd seroprevalence in the region (40 to $70 \%$ ), except in Morocco, which adopted four years of mass vaccination (the last was in 2011 in Eastern Morocco) (EFSA, 2015). The project $\mathrm{TCP} / \mathrm{RAB} / 3302$ was set up in 2010, following the emergence of PPR in Morocco in 2008. Indeed, Morocco was probably the last Northern African country to be infected by PPRV, which was first detected in Egypt during the 1980s (Ismail and House, 1990). An outbreak of PPR was later reported in the Nile delta in 2006 (Abd et al., 2010) and the phylogenetic analyses revealed that the circulating strain of PPRV belonged to lineage IV and was closely related to PPRV isolated in Morocco in 2008 (Kwiatek et al., 2011). Moreover, serological evidence of PPRV infection was observed in Tunisia in small ruminant samples collected in 2006 (Ayari-Fakhfakh et al., 2011). On the other hand, retrospective surveys on a Moroccan serological bank could not detect PPRV antibodies in small ruminant sera collected before 2008 (Ettair, 2012). The results of the molecular studies in each country show that the lineage IV of PPRV is circulating throughout the sub-region (Abd et al., 2010; De Nardi et al., 2012; Sghaier et al., 2014; Kardjadj et al., 2015a) except Mauritania, where El Arbi et al. (2014) reported the presence of the lineage II, therefore, highlighting the existence of a second lineage circulating in North Africa.

These findings stress the importance of an epidemiological survey at a national level to establish the status of the disease in Algeria and to recommend an adequate control strategy. Recently, Kardjadj et al. (2015b) described an overall PPR apparent flock seroprevalence of $42.66 \%(64 / 150)$ and showed a relatively uniform distribution of PPR seroprevalence among all Algerian regions, suggesting a widespread distribution and endemic establishment of PPR in Algerian small ruminant population. Subsequently, in September 2013, the Algerian Veterinary Authority proceeded for the first time with PPR vaccination in Ghardaïa and its neighboring districts (Laghouat, Adrar and El Bayadh) using the vaccine strain Nig.75/1 to avoid an endemic state of the disease in the area.

\section{CONCLUSION}

In recent years, molecular tools have been of tremendous advantage in allowing diagnosis and characterisation of transboundary animal diseases agents in Algeria with far greater accuracy than conventional diagnostic tools. The superior accuracy of the diagnostic tests invariably results in a higher degree of confidence for epidemiological statistics. Molecular tools have also contributed to the identification of origin that may influence the occurrence, the severity of disease and help in the choice of the adequate vaccine for control and possible eradication of TADs from the country.

\section{Conflicts of Interests}

The authors have not declared any conflict of interests. 


\section{REFERENCES}

Abd E-RI, Sharawi S, Barakat M, El-Nahas E (2010). An outbreak of peste des petits ruminants in migratory flocks of sheep and goats in Egypt in 2006. Revue scientifiqueet technique (International Office of Epizootics), 29:655-662.

Ayari-Fakhfakh E, Ghram A, Bouattour A, Larbi I, Gribâa-Dridi L, Kwiatek O, Bouloy $M$, Libeau G, Albina $E$ and Cêtre-Sossah $C$ (2011). First serological investigation of peste-des-petits-ruminants and Rift Valley fever in Tunisia. Vet. J. 187:402-404.

Cêtre-Sossah C, Madani H, Sailleau C, Nomikou K, Sadaoui H, Zientara S (2011). Molecular epidemiology of bluetongue virus serotype 1 isolated in 2006 from Algeria. Res. Vet. Sci. 91(3):486497.

De Nardi M, Lamin Saleh SM, Batten C, Oura C, Di Nardo A, Rossi D (2012). First evidence of peste des petits ruminants (PPR) virus circulation in Algeria (Sahrawiterritories): outbreak investigation and virus lineage identification. Transbound. Emerg. Dis. 59:214-222.

El Arbi AS, El Mamy AB, Salami H, Isselmou E, Kwiatek O, Libeau G, Kane Y, Lancelot R (2014). Peste des Petits Ruminants Virus, Mauritania. Emerg. Infect. Dis. 20:334-335.

Ettair M (2012). Stratégie de surveillance et de lutte contre la PPR au Maroc. Proceedings of the REMESA: atelier conjoint REPIVETRESEPSA des 12 et 13 Juillet 2012, Tunis, 2012.

European Food Safety Authority EFSA, AHAW Panel (2015). Panel on Animal Health and Welfare. Scientific Opinion on peste des petits ruminants EFSA Journal;13(1):3985, 94 p.

FAO (1999). The 1999 Session of the Research Group of the Standing Technical Committee of EuFMD. Available at http://www.fao.org/ag/againfo/commissions/eufmd/

commissions/eufmd-home/reports/archive/ 63rd-session-of-theexecutive-committee/ session-of-the-research-group-of-thestandingtechnical- committee-of-the-eufmd (Access date 09 September 2016).

FAO (2009). Peste des petits ruminants: an increasing threat to small ruminant production in Africa and Asia. EMPRES Transb. Anim. Dis. Bulletin p33.

Grenfell BT, Pybus OG, Gog JR, Wood JL, Daly JM, Mumford JA (2004). Unifying the epidemiological and evolutionary dynamics of pathogens. Science. 303(5656):327-332.

Hamida B. (2000). Les pays touchés par la fièvre catarrhale. 67th General Session of the International Committee of the Office International des Épizooties (OIE). OIE, Paris.

Ismail IM, House J (1990). Evidence of identification of peste des petits ruminants from goats in Egypt. Archiv fur experimentelle Veterinarmedizin, 44:471-474.

Kardjadj M, Ben-Mahdi MH, Pam DL (2015a). First serological and molecular evidence of PPRV occurrence in Ghardaïa district, center of Algeria. Tropical Animal Health \& Production. Online publication May 2015.

Kardjadj M, Metref D, Kouidri B, Pam DL, Ben-Mahdi, M. H (2015b). Seroprevalence, distribution and risk factor for Peste des Petits Ruminants (PPR) in Algeria. Preventive Veterinary Medicine. Online publication September 2015.

Kardjadj M, Pam DL, Ben-Mahdi MH (2016). Sero-epidemiology of bluetongue in Algerian ruminants. African Journal of Biotechnology. Publication May 2016.

Kwiatek O, Ali YH, Saeed IK, Khalafalla AI, Mohamed OI, Obeida AA, Abdelrahman MB, Osman HM, Taha KM, Abbas Z, El Harrak M, Lhor Y, Diallo A, Lancelot R, Albina E and Libeau G (2011). Asian lineage of peste des petits ruminants virus, Africa. Emerg. Infect. Dis. 17:1223-1231.

Levin BR, Lipsitch M, Bonhoeffer S (1999). Population biology, evolution, and infectious disease: convergence and synthesis. Science; 283(5403):806-809.
Maan S, Samuel AR, Maan NS, Attoui H, Rao S, Mertens PP (2009). Molecular epidemiology of bluetongue viruses from disease outbreaks in the Mediterranean basin. Vet. Ital. 40:489-496.

Madani H, Casal J, Alba A, Allepuz A, Cêtre-Sossah C, Hafsi L, Napp S (2011). Animal Diseases Caused by Orbiviruses, Algeria. Emerg. Infect. Dis. 17:2325-2327.

MADR (2015). Ministère de l'Agriculture et de Développement Rural, Algérie. annuel rapport 2015.

Maslow JN, Mulligan ME, Arbeit RD (1993). Molecular epidemiology: application of contemporary techniques to the typing of microorganisms. Clin. Infect. Dis. 17(2):153-162.

Mellor PS, Carpenter S, Harrup L, Baylis M, Mertens PPC (2008). Bluetongue in Europe and the Mediterranean basin: history of occurrence prior to 2006. Prev. Vet. Med. 87:4-20.

OIE (2016). World Animal Health Information Database (WAHID). Available at http://www.oie.int/wahis_2/public/wahid.php/Wahidhome/Home/index content/newlang/en. (Access date 09 September 2016)

Oueslati A, (2012). Mouvements transfrontaliers d'animaux et produits d'origine animale dans les pays REMESA. Proceedings of the REPIVET-RESEPSA workshop, Rome in July 2012.

Riley LW (2004). Molecular epidemiology of infectious diseases. Principles and practices. Washington, DC: ASM Press.

Saegerman C, Berkvens D, Mellor PS (2008). Bluetongue epidemiology in the European Union. Emerg. Infect. Dis. 14:539-544.

Samuel AR, Knowles NJ (2001). Foot-and mouth disease type O viruses exhibit genetically and geographically distinct evolutionary lineages (topotypes). J. Gen. Virol. 82:609-621.

Samuel AR, Knowles NJ, MacKay DKJ (1999). Genetic analysis of type $O$ viruses responsible for epidemics of foot-and-mouth disease in North Africa. Epidemiol. Infect.122:529-538.

Sghaier S, Cosseddu GM, Ben Hassen S, S., Haj Ammar H, Petrini A, Monaco F (2014). Peste des Petits Ruminants Virus, Tunisia, 20122013. Emerg. Infect. Dis. 20:2184-2185.

Thompson RC, Constantine CC, Morgan UM (1998). Overview and significance of molecular methods: what role for molecular epidemiology? Parasitology 117:S161-S175.

Thomson-Aidaros A (2002). Regional status and approaches to control and eradication of Foot and- mouth disease in the Middle East and North Africa. Rev. Sci. Technol. 21:451-458.

Van Belkum A, Tassios PT, Dijkshoorn L, Haeggman S, Cookson B, Fry NK, (2007). Guidelines for the validation and application of typing methods for use in bacterial epidemiology. Clin. Microbiol. Infect.13(3):1-46.

WRLFMD (2016). Molecular Epidemiology/Genotyping, FMD Reference Laboratory Network Reports. Available at http://www.wrlfmd.org/fmd_genotyping/2016. (Access date 09 September 2016). 